\title{
A-MONOTONICITY AND APPLICATIONS TO NONLINEAR VARIATIONAL INCLUSION PROBLEMS
}

\author{
RAM U. VERMA
}

Received 2 March 2004

A new notion of the $A$-monotonicity is introduced, which generalizes the $H$-monotonicity. Since the $A$-monotonicity originates from hemivariational inequalities, and hemivariational inequalities are connected with nonconvex energy functions, it turns out to be a useful tool proving the existence of solutions of nonconvex constrained problems as well.

Recently, Fang and Huang [1] introduced a new class of mappings- $h$-monotone mappings - in the context of solving a system of variational inclusions involving a combination of $h$-monotone and strongly monotone mappings based on the resolvent operator technique. The notion of the h-monotonicity has revitalized the theory of maximal monotone mappings in several directions, especially in the domain of applications. Here we announce the notion of the A-monotone mappings and its applications to the solvability of systems of nonlinear variational inclusions. The class of the A-monotone mappings generalizes the $h$-monotonicity. On the top of that, A-monotonicity originates from hemivariational inequalities, and emerges as a major contributor to the solvability of nonlinear variational problems on nonconvex settings. As a matter of fact, some nice examples on A-monotone (or generalized maximal monotone) mappings can be found in Naniewicz and Panagiotopoulos [2] and Verma [4]. Hemivariational inequalities-initiated and developed by Panagiotopoulos [3] — are connected with nonconvex energy functions and turned out to be useful tools proving the existence of solutions of nonconvex constrained problems. We note that the A-monotonicity is defined in terms of relaxed monotone mappings - a more general notion than the monotonicity/strong monotonicity-which gives a significant edge over the $h$-monotonicity.

Definition 1 [1]. Let $h: H \rightarrow H$ and $M: H \rightarrow 2^{H}$ be any two mappings on $H$. The map $M$ is said to be $h$-monotone if $M$ is monotone and $(h+\rho M)(H)=H$ holds for $\rho>0$. This is equivalent to stating that $M$ is $h$-monotone if $M$ is monotone and $(h+\rho M)$ is maximal monotone. 
Let $X$ denote a reflexive Banach space and $X^{*}$ its dual. Inspired by $[2,4]$, we introduce the notion of the A-monotonicity as follows.

Definition 2. Let $A: X \rightarrow X^{*}$ and $M: X \rightarrow 2^{X^{*}}$ be any mappings on $X$. The map $M$ is said to be $A$-monotone if $M$ is $m$-relaxed monotone and $(A+\rho M)$ is maximal monotone for $\rho>0$.

Lemma 3. Let $A: H \rightarrow H$ be $r$-strongly monotone and $M: H \rightarrow 2^{H}$ be A-monotone. Then the resolvent operator $J_{A, M}^{\rho}: H \rightarrow H$ is $(1 /(r-\rho m))$-Lipschitz continuous for $0<\rho<r / m$.

Example 4 [2, Lemma 7.11]. Let $A: X \rightarrow X^{*}$ be $(m)$-strongly monotone and $f: X \rightarrow R$ be locally Lipschitz such that $\partial f$ is $(\alpha)$-relaxed monotone. Then $\partial f$ is $A$-monotone, that is, $A+\partial f$ is maximal monotone for $m-\alpha>0$, where $m, \alpha>0$.

Example 5 [4, Theorem 4.1]. Let $A: X \rightarrow X^{*}$ be $(m)$-strongly monotone and let $B: X \rightarrow$ $X^{*}$ be $(c)$-strongly Lipschitz continuous. Let $f: X \rightarrow R$ be locally Lipschitz such that $\partial f$ is $(\alpha)$-relaxed monotone. Then $\partial f$ is $(A-B)$-monotone.

Let $H_{1}$ and $H_{2}$ be two real Hilbert spaces and $K_{1}$ and $K_{2}$, respectively, be nonempty closed convex subsets of $H_{1}$ and $H_{2}$. Let $A: H_{1} \rightarrow H_{1}, B: H_{2} \rightarrow H_{2}, M: H_{1} \rightarrow 2^{H_{1}}$, and $N: H_{2} \rightarrow 2^{H_{2}}$ be nonlinear mappings. Let $S: H_{1} \times H_{2} \rightarrow H_{1}$ and $T: H_{1} \times H_{2} \rightarrow H_{2}$ be any two multivalued mappings. Then the problem of finding $(a, b) \in H_{1} \times H_{2}$ such that

$$
0 \in S(a, b)+M(a), \quad 0 \in T(a, b)+N(b)
$$

is called the system of nonlinear variational inclusion (SNVI) problems.

When $M(x)=\partial_{K_{1}}(x)$ and $N(y)=\partial_{K_{2}}(y)$ for all $x \in K_{1}$ and $y \in K_{2}$, where $K_{1}$ and $K_{2}$, respectively, are nonempty closed convex subsets of $H_{1}$ and $H_{2}$, and $\partial_{K_{1}}$ and $\partial_{K_{2}}$ denote indicator functions of $K_{1}$ and $K_{2}$, respectively, the SNVI (1) reduces to determine an element $(a, b) \in K_{1} \times K_{2}$ such that

$$
\begin{aligned}
\langle S(a, b), x-a\rangle \geq 0 & \forall x \in K_{1}, \\
\langle T(a, b), y-b\rangle \geq 0 & \forall y \in K_{2} .
\end{aligned}
$$

Lemma 6. Let $H_{1}$ and $H_{2}$ be two real Hilbert spaces. Let $A: H_{1} \rightarrow H_{1}$ and $B: H_{2} \rightarrow H_{2}$ be strictly monotone, let $M: H_{1} \rightarrow 2^{H_{1}}$ be A-monotone, and let $N: H_{2} \rightarrow 2^{H_{2}}$ be B-monotone. Let $S: H_{1} \times H_{2} \rightarrow H_{1}$ and $T: H_{1} \times H_{2} \rightarrow H_{2}$ be any two multivalued mappings. Then $a$ given element $(a, b) \in H_{1} \times H_{2}$ is a solution to the SNVI (1) problem if and only if $(a, b)$ satisfies

$$
a=J_{A, M}^{\rho}(A(a)-\rho S(a, b)), \quad b=J_{B, N}^{\eta}(B(b)-\eta T(a, b)) .
$$

Theorem 7. Let $H_{1}$ and $H_{2}$ be two real Hilbert spaces. Let $A: H_{1} \rightarrow H_{1}$ be $\left(r_{1}\right)$-strongly monotone and $\left(\alpha_{1}\right)$-Lipschitz continuous, and let $\mathrm{B}: \mathrm{H}_{2} \rightarrow \mathrm{H}_{2}$ be $\left(r_{2}\right)$-strongly monotone and $\left(\alpha_{2}\right)$-Lipschitz continuous. Let $M: H_{1} \rightarrow 2^{H_{1}}$ be A-monotone and let $N: H_{2} \rightarrow 2^{H_{2}}$ be $B$-monotone. Let $S: H_{1} \times H_{2} \rightarrow H_{1}$ be such that $S(\cdot, y)$ is $(\gamma, r)$-relaxed cocoercive and $(\mu)$ Lipschitz continuous in the first variable and $S(x, \cdot)$ is $(\nu)$-Lipschitz continuous in the second variable for all $(x, y) \in H_{1} \times H_{2}$. Let $T: H_{1} \times H_{2} \rightarrow H_{2}$ be such that $T(u, \cdot)$ is $(\lambda, s)$-relaxed 
cocoercive and $(\beta)$-Lipschitz continuous in the second variable and $T(\cdot, v)$ is $(\tau)$-Lipschitz continuous in the first variable for all $(u, v) \in H_{1} \times H_{2}$. If, in addition, there exist positive constants $\rho$ and $\eta$ such that

$$
\begin{aligned}
& \sqrt{\alpha_{1}-2 \rho r+2 \rho \gamma \mu^{2}+\rho^{2} \mu^{2}}+\eta \tau<r_{1} \\
& \sqrt{\alpha_{2}-2 \eta s+2 \eta \lambda \beta^{2}+\eta^{2} \beta^{2}}+\rho \nu<r_{2}
\end{aligned}
$$

then the SNVI (1) problem has a unique solution.

\section{References}

[1] Y. P. Fang and N. J. Huang, H-monotone operators and system of variational inclusions, Comm. Appl. Nonlinear Anal. 11 (2004), no. 1, 93-101.

[2] Z. Naniewicz and P. D. Panagiotopoulos, Mathematical Theory of Hemivariational Inequalities and Applications, Monographs and Textbooks in Pure and Applied Mathematics, vol. 188, Marcel Dekker, New York, 1995.

[3] P. D. Panagiotopoulos, Hemivariational Inequalities. Applications in Mechanics and Engineering, Springer-Verlag, Berlin, 1993.

[4] R. U. Verma, Nonlinear variational and constrained hemivariational inequalities involving relaxed operators, ZAMM Z. Angew. Math. Mech. 77 (1997), no. 5, 387-391.

Ram U. Verma: International Publications, 5066 Jamieson Drive, Suite B-9, Toledo, OH 43613, USA

E-mail address: rverma@internationalpubls.com 


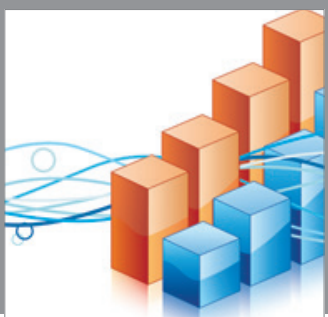

Advances in

Operations Research

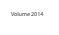

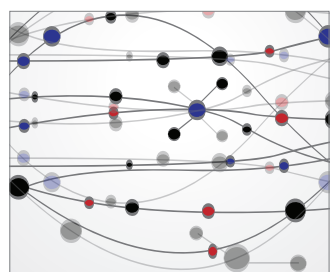

\section{The Scientific} World Journal
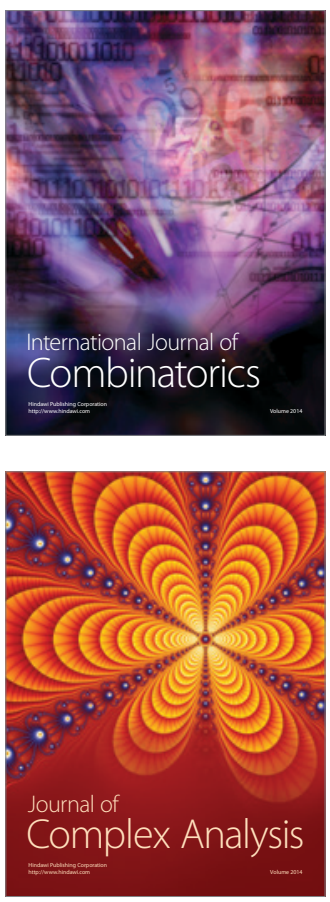

International Journal of

Mathematics and

Mathematical

Sciences
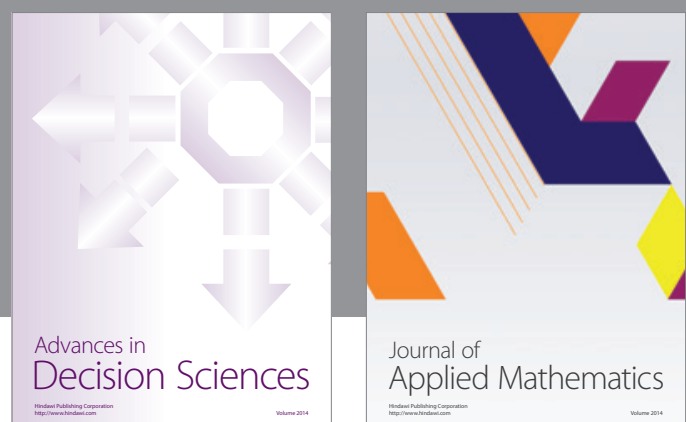

Journal of

Applied Mathematics
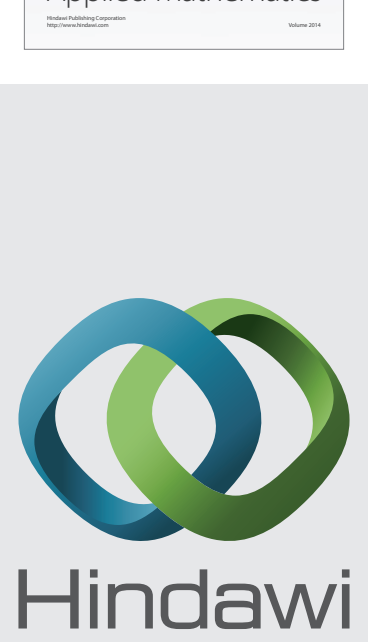

Submit your manuscripts at http://www.hindawi.com
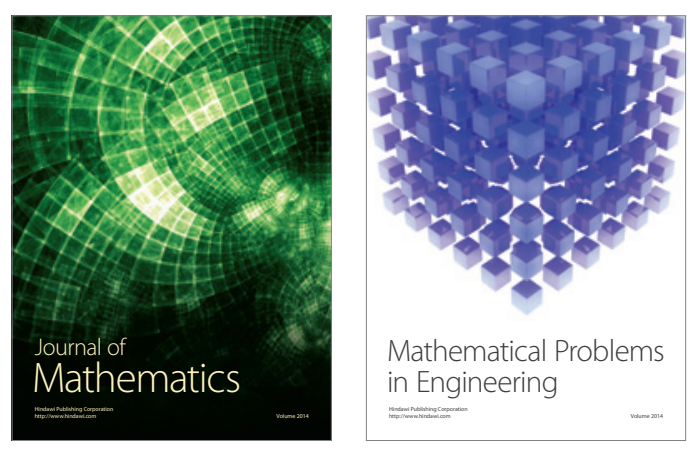

Mathematical Problems in Engineering
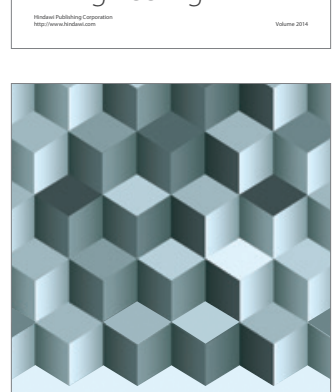

Journal of

Function Spaces
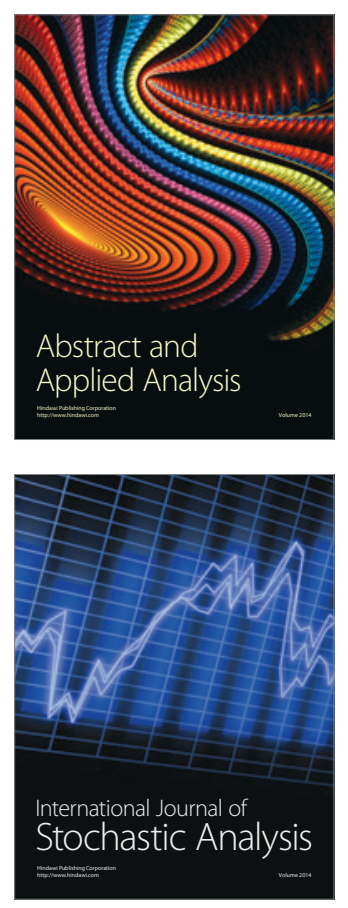

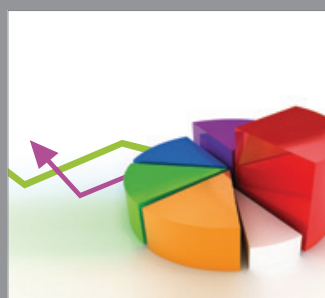

ournal of

Probability and Statistics

Promensencen
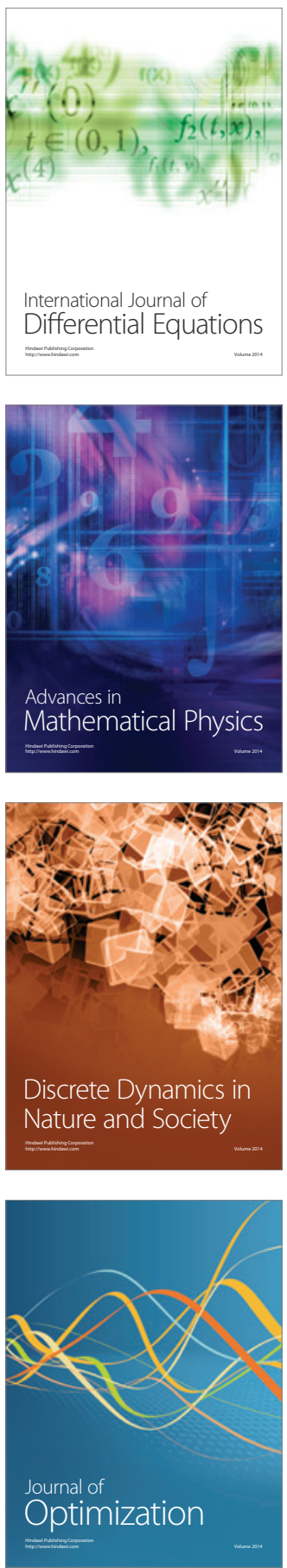